\title{
BAPM: Block Attention Profiling Model for Multi-tab Website Fingerprinting Attacks on Tor
}

\author{
Zhong Guan \\ guanzhong@iie.ac.cn \\ Institute of Information Engineering, \\ Chinese Academy of Sciences \\ School of Cyber Security, University \\ of Chinese Academy of Sciences \\ Beijing, China \\ Zhen Li \\ lizhen@iie.ac.cn \\ Institute of Information Engineering, \\ Chinese Academy of Sciences \\ School of Cyber Security, University \\ of Chinese Academy of Sciences \\ Beijing, China
}

\author{
Gang Xiong \\ xionggang@iie.ac.cn \\ Institute of Information Engineering, \\ Chinese Academy of Sciences \\ School of Cyber Security, University \\ of Chinese Academy of Sciences \\ Beijing, China \\ Mingxin Cui \\ cuimingxin@iie.ac.cn \\ Institute of Information Engineering, \\ Chinese Academy of Sciences \\ School of Cyber Security, University \\ of Chinese Academy of Sciences \\ Beijing, China
}

\author{
Gaopeng Gou \\ gougaopeng@iie.ac.cn \\ Institute of Information Engineering, \\ Chinese Academy of Sciences \\ School of Cyber Security, University \\ of Chinese Academy of Sciences \\ Beijing, China \\ Chang Liu* \\ liuchang@iie.ac.cn \\ Institute of Information Engineering, \\ Chinese Academy of Sciences \\ School of Cyber Security, University \\ of Chinese Academy of Sciences \\ Beijing, China
}

\begin{abstract}
Website fingerprinting attacks on Tor pose an security issue in anonymity privacy, in which attackers can identify websites visited by victims through passively capturing and analyzing encrypted packet traces. Although related works have been studied over a long period, most of them focus on single-tab packet traces which only contain one page tab's data. However, users often open multiple page tabs successively when browsing the web, and multi-tab packet traces generated will corrupt common single-tab attacks. Existing multi-tab attacks still depend on an elaborate feature engineering, besides, they fail to exploit the overlapping area which contains the mixed data of two adjacent page tabs, thus suffering from the information lost or confusion. In this paper, we propose a Block Attention Profiling Model named BAPM as a new multitab attacking model. Specifically, BAPM fully utilizes the whole multi-tab packet trace including the overlapping area to avoid information lost. It generates a tab-aware representation from direction sequences and performs the block division to separate mixed page tabs as clearly as possible, thus relieving the information confusion. Then the attention-based profiling is used to group blocks belonging to the same page tab and finally multiple websites are simultaneously identified under a global view. We compare BAPM with state of the art multi-tab attacks, and BAPM outperforms comparison methods even with larger overlapping area. The effectiveness of
\end{abstract}

\footnotetext{
${ }^{*}$ Chang Liu is the corresponding author.

Permission to make digital or hard copies of all or part of this work for personal or classroom use is granted without fee provided that copies are not made or distributed for profit or commercial advantage and that copies bear this notice and the full citation on the first page. Copyrights for components of this work owned by others than ACM must be honored. Abstracting with credit is permitted. To copy otherwise, or republish, to post on servers or to redistribute to lists, requires prior specific permission and/or a fee. Request permissions from permissions@acm.org.

ACSAC '21, December 6-10, 2021, Virtual Event, USA

(c) 2021 Association for Computing Machinery.

ACM ISBN 978-1-4503-8579-4/21/12 \$15.00

https://doi.org/10.1145/3485832.3485891
}

model design is also validated through ablation, sensitivity and generalization analysis.

\section{CCS CONCEPTS}

- Security and privacy $\rightarrow$ Pseudonymity, anonymity and untraceability.

\section{KEYWORDS}

multi-tab website fingerprinting attack, multi-head attention, deep learning, anonymity

\section{ACM Reference Format:}

Zhong Guan, Gang Xiong, Gaopeng Gou, Zhen Li, Mingxin Cui, and Chang Liu. 2021. BAPM: Block Attention Profiling Model for Multi-tab Website Fingerprinting Attacks on Tor. In Annual Computer Security Applications Conference (ACSAC '21), December 6-10, 2021, Virtual Event, USA. ACM, New York, NY, USA, 12 pages. https://doi.org/10.1145/3485832.3485891

\section{INTRODUCTION}

Concerned about online privacy, Internet users tend to hide their destination websites when browsing the web. For example, as the most widely used anonymous network, the onion routing (Tor) keeps users away from being spied through traffic encryption and random multi-hop routing with volunteer proxy nodes around the world $[1,7]$. However, apart from explicit traffic content and IP addresses, different websites will load unique web resources (e.g., pictures, videos, HTML scripts) when being visited, and some characteristics will be reflected in the external shape of packet traces [12]. Therefore, network administrators are able to identify which websites had been visited by users in the network through analyzing captured packet traces. This security issue is called website fingerprinting (WF) attacks [10].

WF attacks on Tor can be performed on three types of packet traces: TLS packets, TCP packets and cells. Attacks on TLS or TCP packets mainly depend on raw features manually constructed by fine-grained feature engineering $[4,9,12,15,16,24,25]$ such as 
data amount, packet timing, packet length and ordering information. Cells are data transmission units of Tor and all cells have the same length in order to avoid leaking packet length features of specific websites. Therefore, WF attacks on cells actually analyze their direction and deep learning techniques [2, 3, 18-20, 23] are more often used to automatically learn latent features on direction sequences. Both raw and latent features have been proved to be effective, which demonstrates that limited external information can also fingerprint websites.

Although WF attacks on Tor have achieved expected results, their practical feasibility in real world is criticized $[11,26]$. Traditional WF attacks are performed on a well constructed dataset, in which each packet trace is generated by visiting only one page tab at a time. Thus attackers only need to analyze traces entirely belonging to a specific website without any noise data, which is known as singletab assumption. This assumption makes WF attacks less difficult, but it is obviously unreal: Users may do other things such as instant messaging along with web browsing, and similar behavior is totally random [26]. In addition, even if users only browse the web, they may open a new page tab before the last one finishes loading which is called multi-tab browsing behavior [6, 8, 11, 26, 29], for example, jumping to another page through a hyperlink. According to [22], about $78 \%$ of the browsing time has at least two parallel opening page tabs for one user, so packet traces in real world inevitably contain mixed data from multiple page tabs and greatly degrade common single-tab WF attacks.

\begin{tabular}{|c|c|c|c|}
\hline Website A & Idle time & Website B & (a) Apart traces \\
\hline \multicolumn{2}{|c|}{ Website A } & Website B & (b) Continuous traces \\
\hline Website A & Overlapping area & Website B & (c) Overlapping traces \\
\hline
\end{tabular}

\section{Figure 1: Three basic situations in two-tab packet traces}

According to [26], multi-tab packet traces have three basic types: apart, continuous and overlapping ones. Fig. 1 displays three situations of two-tab traces as an example. Apart and continuous traces are easier to process since data of each page tab is independent, while overlapping traces are much more complicated and become a critical issue in multi-tab WF attacks. It is almost impossible to accurately separate two overlapping page tabs mixed in one trace, changeable overlapping area size and page number also bring much uncertainty. In this paper, we focus on the multi-tab WF attacks for overlapping traces like Fig. 1(c) of Tor.

Multi-tab WF attacks are put forward in recent years $[6,8,26$, $29,30]$ to relax the single-tab assumption, among them attacks on overlapping traces include two types: (1) One type ignores the overlapping area. It is performed in two steps which determines the range of overlapping area and drops it in advance, then exploits remaining part of traces with only one page tab's data. (2) Another type utilizes the whole multi-tab trace including overlapping and non-overlapping area. Except for cited works, we also regard the multi-label classification in machine learning as an attacking method of this type, since it can learn multiple labels directly from one sample. All single-tab WF attacks can be customized for multitab scenarios using multi-label classification theoretically, although it has not been implemented in any related works. These two methods have gained important achievements, but there are still some remaining problems to be solved:

- Expensive manual cost. Existing multi-tab WF attacks all depend on a fine-grained feature engineering. A well designed feature set often requires attackers to put in a lot of effort. Besides, methods ignoring the overlapping area even need to extract features two times for splitting point and non-overlapping area respectively.

- Insufficient overlapping area exploiting. Both two types of multi-tab WF attacks have their own problem facing the overlapping area: (1) Information lost. Given that multi-tab traces in real world can have a large overlapping area, simply discarding this area will ignore much useful information and thus restricted by a limited view. (2) Information confusion. Since the overlapping area is mixed by many discontinuous parts of two or more pages, directly taking the overlapping area into consideration without distinguishing different parts within it can result in a worse effect.

In this paper, we propose a Block Attention Profiling Model BAPM as a multi-tab WF attack based on deep learning. BAPM leverages the $\mathrm{CNN}$ to generate a tab-aware representation on the whole packet trace including overlapping area, and splits the representation into blocks to reduce the influence of mixed data. A series of attention scores are then calculated on blocks according to block relations, therefore blocks of the same website are grouped as an integral part to mutually make a contribution on the corresponding website identification. As a result, BAPM preserves the overlapping area and well exploits it rather than being confused. Through this work, we reveal potential privacy threats of multi-tab WF attacks. Main contributions of this paper are listed below:

- BAPM is the first end-to-end model for multi-tab WF attacks: (1) It generates the tab-aware representations without feature engineering. (2) It performs the attack on the whole multi-tab packet trace to avoid information lost.

- We relieve the information confusion by designing a block division on tab-aware representations. We try to ensure that the trace part of each block only represents one website, thus blocks will not be confused by mix data.

- We import multi-head attention to group blocks which have stronger relations. Block groups provide us a global view to adaptively profile multi-tab traces: blocks in this group will be all focused when identifying the corresponding website, no matter where is it located in the original trace.

- We compare BAPM with three multi-tab WF attacks on public and self-built dataset, results show that BAPM has the best and most stable performance. Effectiveness of BAPM components is also validated.

The rest of this paper is structured as follows. We summarize related single-tab and multi-tab WF attacks in section 2 and describe the threat model in section 3. BAPM is proposed with three modules in section 4 . We introduce the dataset, experiment settings and design in section 5. Section 6 analyzes experiment results and section 7 discusses model performance in real world. We conclude this paper and provide possible future works in section 8 . 


\section{RELATED WORK}

\subsection{Single-tab WF Attacks}

Traditional WF attacks are based on the single-tab assumption, including attacks on non-Tor and Tor scenarios.

Attacks on non-Tor: Single-tab WF attacks are firstly proposed by Hintz [10], he determined if the user is visiting certain websites through data amount in bytes received on specific ports. Since then, similar methods are widely applied in encrypted traffic analysis. Liberatore et al. used the direction and size of packets to profile websites behind OpenSSH and identify them based on these profiles [13]. Lu et al. exploited packet ordering information along with packet lengths of websites on both SSH and SSL tunnels [14].

Attacks on Tor: In 2011, Panchenko et al. successfully performed WF attack on Tor for the first time [16], showing that the onion routing based system is not as strong as expected. WF attacks on Tor at early stage are mainly machine learning models like KNN [24], random forest [9], SVM [25] and improved SVM [4, 15]. Features extracted for attacking also have a rich variety: Panchenko et al. generated a more efficient cumulative representation of packets ordering and bursts [15], Li et al. discussed in detail how different feature sets leak website information [12].

Deep learning based methods are firstly used on Tor by Abe [2], he adopted stacked auto-encoders and results on simple direction sequences are comparable to feature based methods. Afterwards, results of deep learning models keep getting better and better with more complex structures. Rimmer et al. proved that deep learning approaches are more robust against website content changes, showing the feasibility of automated feature learning [18]. Sirinam et al. proposed an elaborate CNN structure called Deep Fingerprinting (DF) [19]. DF expanded the operations in one basic convolutional block and repeated it four times, attaining $98.3 \%$ accuracy on close world. Bhat et al. presented Var-CNN with semi-automated featrue extraction [3] and achieved better results under small amounts of training data.

\subsection{Multi-tab WF Attacks}

Multi-tab WF attacks have also been performed on both non-Tor and Tor traffic with different ways.

Attacks on non-Tor: Naive bayes classifier [8], hidden markov model [30] and recurrent neural network [17] are used to attack multi-tab browsing behavior based on specific protocol features. For example, [8] gets the TCP connection number as the key information by counting the open channel message of SSH, while [17] depends on the server name field of TLS client hello messages.

Attacks on Tor: Multi-tab WF attacks on Tor are considered in practice by Juarez et al. for the first time [11], and the accuracy dropped significantly. In 2016, Wang et al. analyzed all three multitab conditions. They handled the apart and continuous packet traces well through time-based and classification-based splitting, but failed with overlapping ones.

Page splitting [29] and page sectioning [6] WF attacks (denoted as PSP-WF and PSE-WF) are two latest works on overlapping traces. They represent two types of multi-tab WF attacks introduced above. PSP-WF firstly finds and removes the overlapping area by pinpointing the boundary packet of it called splitting point. Since an overlapping trace has only two splitting points (at the begin and end of overlapping area) but much more other packets, [29] handles the imbalance through a balance-cascade classifier to selectively drop normal packets during the model training iterations. Once the splitting point is pinpointed, the part of trace before the first point and after the second point would be input to a random forest classifier to get corresponding website label. PSP-WF involves two classification tasks but can only classify the first page [29]. Besides, attackers need to record the splitting point location when building the training set.

PSE-WF do not particularly single the overlapping area out. It divides the entire trace into several sections which have the same packet number or time length, and represent each section with CUMUL features [15]. The model training and testing are all based on the section as a unit: sections of single-tab traces are used to train a K-NN classifier, while sections of multi-tab traces are used to test and predict each section label. Finally the trace label will be determined by the majority voting results of all section labels. However, testing sections in overlapping area are more likely to be wrongly classified and negatively affect the voting results instead of providing valid information in overlapping area, thus PSE-WF requires a limited overlapping size in particular [6].

Same as PSP-WF and PSE-WF, BAPM is a multi-tab WF attack on overlapping traces of Tor, more details about comparison between these two works and BAPM are displayed in Table 1.

\section{THREAT MODEL}

Model assumption: A critical step in WF attacks is how to capture victims' packet traces for prediction. There are two types of attackers who have access to raw packet traces: observers and forwarders. Observers monitor all packets received or sent by a network such as Internet service provider on the backbone network. Forwarders have a stronger capability which can communicate with users like a proxy node, but it is obviously harder to act as a malicious middleman than only monitoring from a third-person perspective. For example, observers in Tor only see encrypted packet traces with no extra information, but forwarders can negotiate with users to establish a anonymous circuit to transmit data. During the transmission, forwarders can obtain cell logs of all packets going through their node by modifying the protocol source code. Since each session has a unique circuit identifier which is recorded in cell log, forwarders can separate overlapping traces by checking the circuit identifier. So multi-tab browsing behavior makes no difference for forwarders actually, and multi-tab WF techniques discussed here is designed for observers rather than forwarders.

There are also some other mutual constrictions in multi-tab WF attacks. Firstly, background noise is not considered, all packet traces are only generated by the web browsing. In addition, each website is represented by its home page, thus the website classification actually refers to classifying web pages instead of the website family with a series of sub-pages. Note that these constrictions are also followed by other multi-tab WF attacks, so the capabilities of our model are not extra enhanced.

Model overview: A typical case of multi-tab WF attacks is shown as Fig. 2. Similar to single-tab attacks, attackers maintain a list of websites they are interested to monitor, and visit these websites by themselves to collect enough packet traces for each website. 
Table 1: Characteristics comparison between BAPM and state of the art multi-tab WF attacks

\begin{tabular}{c|ccc}
\hline & PSP-WF [29] & PSE-WF [6] & BAPM \\
\hline Model Type & Feature engineering based & Feature engineering based & End-to-end \\
Data Type & Real world & Manually merged & Real world \& Manually merged \\
Model Capability & For the first page & For two pages & For more than two pages \\
Prior Knowledge & Splitting point location \& Page number & Page number & Page number \\
Model Retrain & When overlapping ratio changes & No need & No need \\
Overlapping area size & More than 50\% of traces & Around 20\% of traces & More than 50\% of traces \\
\hline
\end{tabular}

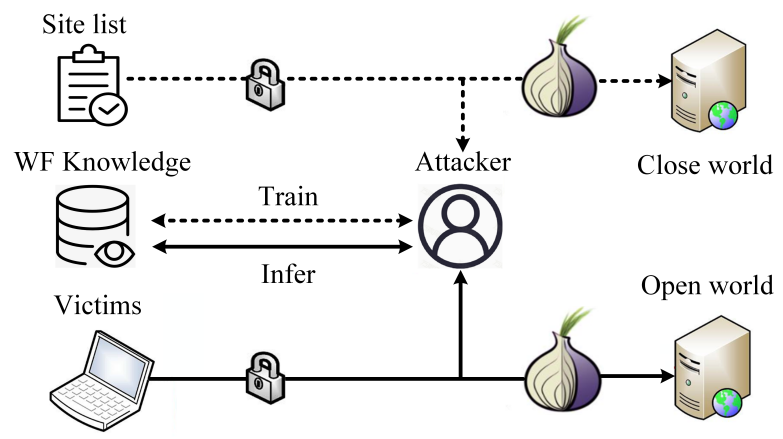

Figure 2: Threat model for WF attacks on Tor

Then they train a WF classifier using features of collected traces, the classifier input can be raw sequence features (e.g., bytes, time and direction sequence) or high-level features reorganized by statistical methods (e.g., standard deviation of raw features). A well trained classifier can correctly map the features to corresponding website labels, thus attackers can infer the websites visited by victims through extracting features from their packet traces in the same way. As equation 1 shows, $W$ is a set of monitored websites, and $w$ represents a list of website labels in a multi-tab packet trace. The used feature here $f$ is the direction sequence in which cells from client to server is recorded as 1 and otherwise -1 . The function classifier stores the WF knowledge on monitored websites.

$$
w=\text { Classifier }\left(\left[f_{1}, f_{2}, \ldots, f_{n}\right]\right), f_{i} \in\{1,-1\}, w \in W, w \geq 2
$$

In fact, victims may visit those websites which do not appear on the attacker's list. This situation is called open world. On the contrary, close world indicates that victims only visit those websites which have already been analyzed by WF classifiers. Open world can be seen as the special case of close world with one more website class, and this unknown class has the highest proportion among all classes because it includes all packet traces of websites ignored by attackers. Thus open world is often used to examine the robustness of WF classifiers in real world.

\section{ARCHITECTURE OF BLOCK ATTENTION PROFILING MODEL}

BAPM has three main parts shown as Fig. 3. Tab-aware representation and block division are responsible for separating overlapping websites in multi-tab traces, and attention-based profiling groups separated trace parts.

\subsection{Tab-aware Representation}

Attackers collect multi-tab packet traces generated when successively browsing A.com, B.com or more site pages through anonymous network. Collected traces are then transformed into cell sequences with direction. All direction sequences have the same maximum length by truncation or padding with 0 . Next we let the model automatically learn a latent feature representation from direction sequences through a convolutional neural network. The network has three one-dimensional convolution layers which are adaptive to deal with direction sequences since they have the same dimension number. Each layer has four types of operation including convolution, activation, batch normalization and max pooling. As shown in equation 2 , one direction sequence $\left[f_{1}, \ldots, f_{n}\right]$ is abstracted into a set of feature vectors as the high-level representation in first layer, new vectors are processed by the same way and more shorter feature vectors are generated in the second layer, then the third layer. Finally we get a two-dimensional feature map $F$ with $r \times c$ elements $v_{i j}$.

$$
F=\operatorname{convolution}\left(\left[f_{1}, \ldots, f_{n}\right]\right), f_{i} \in\{1,-1\}, F=\left(v_{i j}\right)_{r \times c}
$$

The feature map is next concatenated as a one-dimensional tabaware representation. The concatenation follows the direction of column axis instead of merging rows in feature map end to end. As shown in Fig. 4, assume that feature vectors output by the last convolution layer are $\left(x_{11}, x_{12}, x_{13} \ldots\right),\left(x_{21}, x_{22}, y_{21} \ldots\right) \ldots\left(x_{n 1}, y_{n 1}\right.$, $\left.y_{n 2} \ldots\right), x$ and $y$ denote two websites while the number indexes identify vector elements. Two vectors are merged by columns as arrows indicate, forming the vector $\left(x_{11}, x_{21} \ldots x_{n 1}, x_{12} \ldots\right)$ which is in accordance with original positions of website $x$ and $y$ in raw multi-tab packet traces.

New representation preserves the original order of page tabs browsed by users, also together with potential information hidden in these tabs, thus we call it a tab-aware representation. Besides, different parts within the same website can also inherit the order consistency. Tab-aware representation take the whole packet trace into account including overlapping and non-overlapping area to enrich the WF attacking model knowledge and separates multiple websites as clearly as possible in the tab level, thus providing convenience for following operations. 

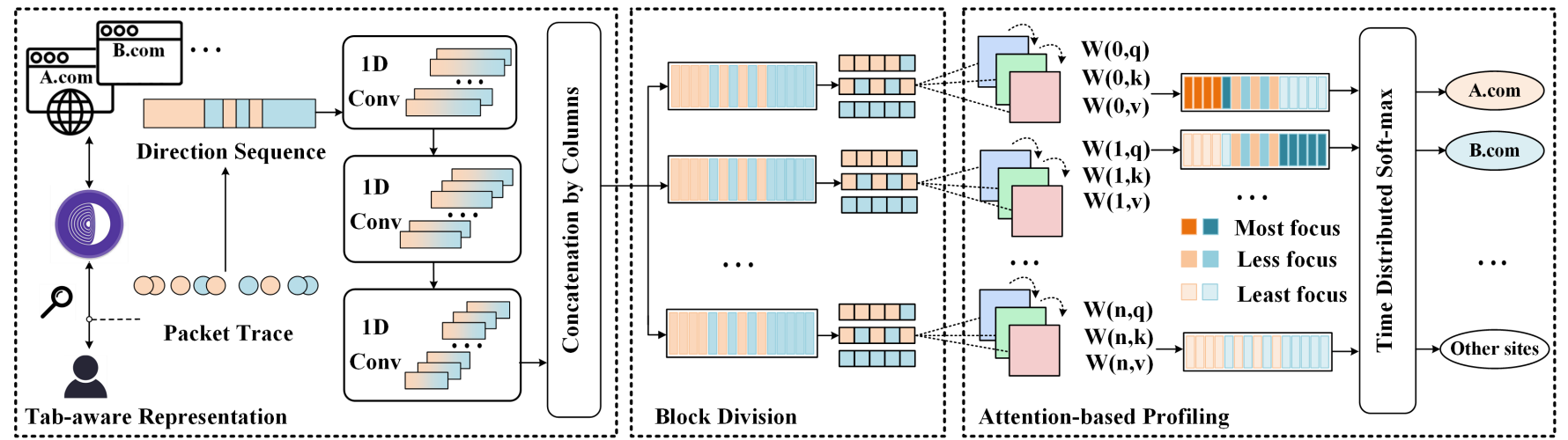

Figure 3: The architecture of Block Attention Profiling Model BAPM

\subsection{Block Division}

Making use of the overlapping area effectively is important for multi-tab attacks, but it is confusing with mixed data although a tab-level order of websites is maintained in tab-aware representations. To further separate websites in overlapping area, we divide tab-aware representations into several blocks and try to discover relations among blocks. The concept of "block" here differs from the section in PSE-WF: one block is a part of feature representation instead of original traces, and we will classify the website after grouping related blocks together instead of depending on the result of one single block. The relations between each block and other blocks will be learned and enhanced during the model training iteration by the following self-attention module. Blocks with stronger relation will contribute on the classification of the same website mutually, while an irrelevant block will be excluded to mitigate the influence of mixed data.

A proper block size needs to be specified to maximize the block division effect. There is not much we can utilize in smaller blocks to be the basis for relation discovery, while larger blocks in overlapping area are as complicated as no division since they are still involved with mixed data. We determine the 16 as the best block number through parameter tuning in section 6.3.

Block division has two advantages: (1) It provides a finer granularity for the attention module to fuse representation segments of the same website, thus optimizing the attention distribution. Attention scores on single values are likely to be wrongly calculated due to poor context information, but the condition would improve if there are other values to be referred within one block, especially when the block consists almost entirely of one website's data. (2) It reduces the model complexity. According to the model design, the size of BAPM can reach up to $37 \mathrm{MB}$ without block division. But this number can be reduced by more than 80 percents under 16 blocks division because attention scores are calculated in blocks instead of a lot more representation elements. Section 6.2 and 6.3 will confirm that through experiments.

\subsection{Attention-based Profiling}

Tab-aware representation blocks can not be directly input to a WF classifier altogether since blocks come from several websites. Therefore, we need to fuse information of blocks belonging to the same website to create some adaptive varieties of original representation, and each variety is adjusted to only highlight one website. As a widely used deep learning technique $[5,21,28]$, the attention mechanism satisfies the requirement by enabling the model to pay attention of different levels to different sample parts.

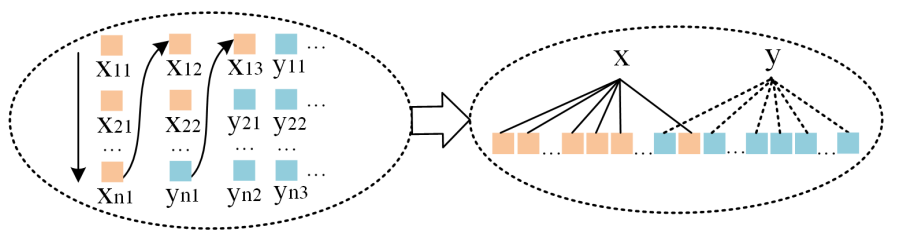

Figure 4: Diagram of concatenation by columns

As shown in equation 3, three types of vectors named query $(Q)$, key $(K)$ and value $(V)$ participate in the attention mechanism. All block vectors of tab-aware representation are stored in $V . K$ can be seen as a series of storage bucket addresses, and each bucket stores an element of $V . Q$ is also an address set, and addresses point to blocks we want to query. Assume that $k, v$ are elements located in the same position of $K, V$ (i.e., the bucket in address $k$ stores the vector $v$ ), and $q$ is the corresponding query address of block $b$. If $K$ and $V$ have n elements, attention mechanism will generate $\mathrm{n}$ values for each block by linking its address $q$ with all bucket address $k_{i}$ and then taking some value (no more than value of vector $v_{i}$ ) out from buckets in address $k_{i}$. Therefore, the attention mechanism can be described as the mapping rules from query-key pairs to key-value pairs [21] as names "query", "key" and "value" imply. The calculation includes following two steps:

$$
\operatorname{Attention}(Q, K, V)=\operatorname{softmax}\left(\frac{Q K^{T}}{\sqrt{d_{k}}}\right) V
$$

(1) The first step is determining how to link a block address $q$ with bucket addresses $k_{i}$. Mainstream methods measure the similarity between $q$ and $k_{i}$ through dot multiply, multi-layer perception or simply concatenating two vectors, which is denoted as $Q K^{T}$. The obtained similarity vectors are divided by the square root of $K$ 's dimension number $\sqrt{d_{k}}$, and then normalized by a softmax 
activation layer. The division and softmax operation all help to minimize the possible deviation in calculation results. The weights vector with sum of 1 output by the softmax layer is exactly attention scores which is positively correlated with the similarity: a higher attention score means that $q$ is enough similar to $k_{i}$, thus the block with address $q$ has a strong relation with the block vector stored in $k_{i}$. On the contrary, if two blocks have a weak relation, the attention score on $q$ and $k_{i}$ should be low. Extend $q$ towards $k_{i}$ to $q_{i}$ towards $k_{i}$, and we can profile the relations between any two blocks in the form of attention scores.

(2) The second step is distributing attention to all blocks according to attention scores. Vectors in $V$ are multiplied by corresponding attention score just like a masking operation, a larger score on the pair $\left(q, k_{i}\right)$ allows the model to take more value out from the block vector $v_{i}$, otherwise it only takes a little or even nothing. All masked vectors form the new tab-aware representation concerned with the block relations. For example, block $b$ belonging to A.com in original representation will be grouped together with other blocks of A.com and their vector values are higher in the representation variety of A.com. On the contrary, value of $b$ will be minimized since it has a weak relation with those blocks of B.com, thus influence of $b$ on B.com classification will be reduced.

Similarly, we repeat the above operation for each website, constructing a multi-head attention structure. As shown in equation 4, attention scores are calculated on each attention head along with the parameter iteration of weight matrices $W$ on each group of $Q, K$, $V$. One head generates one adaptive variety of the same tab-aware representation for a specific website. When the number of websites in multi-tab packet traces changes, the model can be expanded easily with only adjusting the head number. Finally, the output of heads is distributed to their respective softmax layers to classify one of $N$ websites as equation 5 shows. Through attention mechanism, information of different websites is fused together without explicit specifying the website location.

$$
\begin{gathered}
\text { head }_{i}=\operatorname{Attention}\left(Q W_{i}^{Q}, K W_{i}^{K}, V W_{i}^{V}\right) \\
\text { website }_{i}=\operatorname{softmax}\left(\text { head }_{i}\right), i \in[1, N]
\end{gathered}
$$

For example, A.com and B.com are represented by yellow and blue area in Fig. 3 respectively. The upper attention head gives a new representation for A.com after the calculation involved with $W(0, q), W(0, k)$ and $W(0, v)$. Compared with original tab-aware representations output by the concatenation, the new one mostly focuses on the A.com part, secondarily focuses on the overlapping area and least focuses or even pays no attention on remained B.com part. Three parts are marked with the dark, normal and light color respectively. For simplicity, we only display representation varieties including first two websites. Since the bottom head is prepared for the last websites, the A.com and B.com parts in this head are all applied with the light color.

\section{EXPERIMENT SETUP}

\subsection{Experiment Design and Settings}

According to the achieved results and problem relevance of existing WF attacks, PSP-WF [29], PSE-WF [6] and DF [19] are chosen as comparison methods in following experiments. We reproduce the work of PSP-WF and PSE-WF with optimal parameters, and build a new model named Multi-DF by making DF suitable for predicting multiple labels on one packet trace. Specifically, according to the common principle of multi-label classification, we replace the softmax activation function in DF with sigmoid and use the binary entropy loss function instead of categorical one, then we can get multiple website labels according to the maximum probability values. Note that other single-tab WF attacks can also be modified in the same way, and we choose DF since it is the state of the art WF attacking model among them so far.

To comprehensively illustrate the advantages of BAPM, we design experiments from three main aspects: (1) The comparison experiment. We compare four methods under five overlapping proportion ranging from $10 \%$ to $50 \%$ on both the first and the second page in section 6.1. (2) Model design validation including ablation and sensitivity analysis. Ablation analysis in section 6.2 gives the classification results before and after using block division and multi-head attention to prove their effectiveness. Sensitivity analysis in section 6.3 conducts a parameter tuning on the block number to research its influence on model performance. (3) Generalization experiments. We perform experiments when overlapping page tab number increases to 3 in section 6.4 to test the generalization of BAPM. Experiments on the open world dataset when non-monitored websites number is 2000,4000 and 6000 are also considered in section 6.5 .

Table 2 shows crucial parameter settings of three model parts including $\mathrm{CNN}$ architecture parameters, the block number and attention vector dimensions. The settings will be used in above experiments, except for the three-tab experiment in which some vector dimensions need to be extended.

Table 2: Parameter settings of BAPM

\begin{tabular}{c|c|c}
\hline Model Part & Design Details & Value \\
\hline \multirow{3}{*}{ Tab-aware representation } & Input Dimension & $(8192,1)$ \\
& & $(12288,1)^{*}$ \\
& Filter Number & {$[32,64,128]$} \\
& Filter Length & {$[5,5,5]$} \\
& Pooling Size & {$[8,8,8]$} \\
\hline \multirow{2}{*}{ Block division } & Block Number & 16 \\
& Scanning Range & $(1 \leq \mathrm{n} \leq 512)$ \\
\hline \multirow{3}{*}{ Attention-based profiling } & Input Dimension & $(2048,1)$ \\
& & $(3072,1)^{*}$ \\
& Output Dimension & $(256,1)$ \\
& & $(384,1)^{*}$ \\
\hline
\end{tabular}

${ }^{*}$ Extended vector dimensions for the three-tab experiment

We use the accuracy (Acc), precision (Pre) and recall (Rec, also known as true positive rate) rate as evaluation metrics. According to the widely accepted definition, accuracy is the correctly classified proportion in all testing traces. Precision and recall rate are calculated for a certain class like A.com, which are the correctly classified proportion in all testing traces predicted as A.com, and in all testing traces truly belonging to A.com, respectively. We report 
the average precision and recall rate of all 50 website classes when analyzing experiment results.

\subsection{Dataset}

We have three manually merged datasets and one real dataset in our experiments. Manually merged datasets are constructed from a public single-tab dataset in [27]. It contains 10398 websites in total, 108 close world websites of which have around 100 packet traces, and remaining open world websites all have only one packet trace. Each trace has thousands of Tor cells denoted by $1,-1$ for direction and 0 for padding. We choose this dataset because merging multi-tab traces needs to know both cell timestamp and direction of original single-tab traces, and dataset in [27] is the largest one meeting the requirement.

Close world multi-tab dataset. We select 50 websites which have most packet traces in original dataset, 90 traces of each website is used as train set and remaining traces are test set. One two-tab trace is obtained by performing a sorting algorithm on two singletab traces according to packet timestamps like [6]. The beginning packet timestamps of the first and second tab are set to be 0 and a predetermined delay time before the sorting action, then packets with smaller timestamps will be arranged in the front. We merge two traces for each sample of websites to make sure that it appears on both the first and second tab location at least once. The another tab is selected randomly from 50 websites. Finally we have 9000 training traces and 1708 testing traces with "A.com \& other" and "other \& A.com" mode.

Open world multi-tab dataset. We pick out 2000, 4000 and 6000 websites from 10290 open world websites of the original singletab dataset. We still merge two multi-tab traces for each sample of close world websites but another tab is randomly selected from 2050, 4050 and 6050 websites respectively. Thus the open world dataset has the same size with the close world one, but involves open world websites in three scales.

Three-tab dataset. Considering that multi-tab packet traces in reality can have more than 2 overlapping page tabs, we also construct a three-tab dataset to examine our model. We merge three traces for each sample of close-world websites and other two tabs are randomly selected from 50 websites. Finally it has 13500 training traces and 2562 testing traces.

Note that we use the manual instead of real traces as major datasets because (1) We need know the accurate ratio of overlapping area to illustrate the effectiveness of BAPM (e.g., whether the model is stable when the overlapping ratio increases), but we do not know the overlapping ratio of real traces because we can not precisely control when the first tab ends loading. (2) Attackers need to visit two websites 10000 times for capturing 10000 real two-tab traces, but they can only visit A.com and B.com 100 times respectively and then produce 10000 traces through $100^{*} 100$ possible combinations. Thus the manual dataset can more efficiently utilize the collected data. Since above two reasons are reasonable only when manual traces have similar influence on the model with real traces, we also build a real world multi-tab dataset to examine BAPM on real traces we collected in section 7.1. The real world dataset has 10000 training traces and 1000 testing traces involving 50 websites.

\section{EXPERIMENT RESULTS ANALYSIS}

\subsection{Comparison experiment results}

Table 3 shows the overall accuracy, average precision and recall rate of the comparison experiment. Note that we do not retrain BAPM for different overlapping proportion, results on $10 \%-40 \%$ proportion are obtained by BAPM trained on $50 \%$ proportion for one time. We can draw following conclusions:

(1) BAPM achieves the best accuracy around $85 \%$ with five overlapping proportions under all evaluation metrics. The second best method is PSP-WF which reaches the accuracy exceeding $80 \%$ when overlapping proportion is small. The trend of average precision and recall rate are similar to the accuracy, and this situation is also found in following analysis. Results of PSE-WF and Multi-DF are not ideal all the time, according to above theoretical analysis, PSEWF is heavily reliant upon every section label thus it will ignore what is out each section, and sections in overlapping area confuses the majority voting results. Multi-DF directly classifies the whole trace including overlapping area indiscriminately, so it will also be inevitably confused similar to PSP-WF. Another evidence of the confusion is that we test DF on the original single-tab dataset and it reaches $94.73 \%$ accuracy when acting as a single-tab attack. But the accuracy is nearly cut in half when predicting multi-tab traces.

(2) BAPM has a stronger robustness when overlapping area size increases, with a decline no more than 3.2\%. PSE-WF and DF are also stable but it's possibly because their results on small overlapping proportion are already limited. PSP-WF declines most obviously since it will drop the half of one packet trace when overlapping proportion is $50 \%$, and it's difficult to keeping the effectiveness with such a serious information lost.

(3) Benefited from exploiting the overlapping area well, BAPM outperforms other methods by $30 \%$ at least on the second page. According to previous multi-tab WF attack results, the second page is usually harder to distinguish than the first page. [6] illustrates the reason why the second page classification is more challenging is that the beginning part of a packet trace plays a more important role in the classification. However, except for the first page, all beginning parts of following pages are covered by the overlapping area, mixed with its previous page. Consistent with this finding, results on the second page inevitably show a decline, especially for PSP-WF which removes the overlapping area. Besides, PSPWF needs to pinpoint the splitting point firstly but the prediction accuracy drops about $30 \%$ for the second splitting point. Thus the second page is more likely to be wrongly split and influence the next website classification result.

\subsection{Ablation Analysis}

As mentioned above, the block division and attention-based profiling are two key components in BAPM to deal with multi-tab packet traces. We conduct two ablation analysis experiments for them separately to demonstrate their effectiveness. Three comparison models are full BAPM, BAPM without block division and BAPM without multi-head attention. Figure 5(a), Figure 5(b) and Figure 5(c) display three evaluation metrics, and solid lines depict results on the first page while dotted lines represent the second page.

Compare the results between BAPM with and without the block division module, we can find that the block division help to improve 
Table 3: Comparison experiment results on two-tab packet traces under five overlapping proportions

\begin{tabular}{|c|c|c|c|c|c|c|c|c|c|c|c|c|c|c|c|c|}
\hline \multirow{2}{*}{\multicolumn{2}{|c|}{$\frac{\text { Overlapping proportion }}{\text { Metrics }}$}} & \multicolumn{3}{|c|}{$10 \%$} & \multicolumn{3}{|c|}{$20 \%$} & \multicolumn{3}{|c|}{$30 \%$} & \multicolumn{3}{|c|}{$40 \%$} & \multicolumn{3}{|c|}{$50 \%$} \\
\hline & & Acc & Pre & Rec & Acc & Pre & Rec & Acc & Pre & Rec & Acc & Pre & $\operatorname{Rec}$ & Acc & Pre & $\operatorname{Rec}$ \\
\hline \multirow{4}{*}{ 1st Page } & BAPM & 85.1 & 86.1 & 85.0 & 86.2 & 86.8 & 85.9 & 84.7 & 85.1 & 84.5 & 86.4 & 86.8 & 86.4 & 83.2 & 84.0 & 82.9 \\
\hline & PSP-WF & 81.1 & 81.8 & 80.4 & 71.9 & 74.5 & 70.6 & 64.4 & 68.6 & 63.0 & 54.4 & 60.9 & 52.0 & 42.5 & 51.0 & 40.5 \\
\hline & PSE-WF & 36.7 & 57.5 & 36.2 & 35.0 & 52.3 & 34.6 & 38.4 & 56.4 & 37.4 & 36.4 & 54.2 & 35.7 & 37.6 & 56.6 & 36.5 \\
\hline & Multi-DF & 51.1 & 50.0 & 50.6 & 50.9 & 49.7 & 50.9 & 50.5 & 50.2 & 52.0 & 51.9 & 51.4 & 52.7 & 48.7 & 48.4 & 49.5 \\
\hline \multirow{4}{*}{ 2nd Page } & BAPM & 77.9 & 78.7 & 77.9 & 78.9 & 79.3 & 78.8 & 78.0 & 78.4 & 77.2 & 75.0 & 74.6 & 74.4 & 71.9 & 72.5 & 71.3 \\
\hline & PSP-WF & 27.0 & 41.0 & 25.5 & 22.1 & 35.0 & 20.2 & 19.2 & 29.0 & 18.5 & 15.7 & 24.5 & 15.0 & 15.4 & 23.4 & 14.9 \\
\hline & PSE-WF & 30.7 & 47.2 & 29.8 & 30.1 & 46.8 & 29.0 & 31.9 & 49.2 & 30.6 & 30.6 & 48.0 & 29.5 & 29.3 & 48.1 & 28.0 \\
\hline & Multi-DF & 40.8 & 42.6 & 39.0 & 41.9 & 42.6 & 40.2 & 39.1 & 41.2 & 38.7 & 41.5 & 40.9 & 40.3 & 36.0 & 37.2 & 37.8 \\
\hline
\end{tabular}

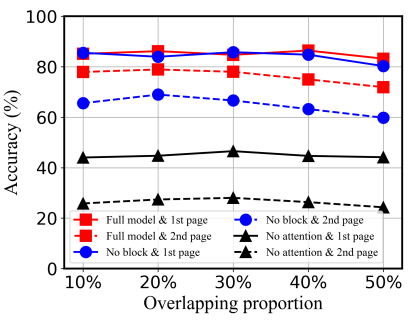

(a) Overall accuracy

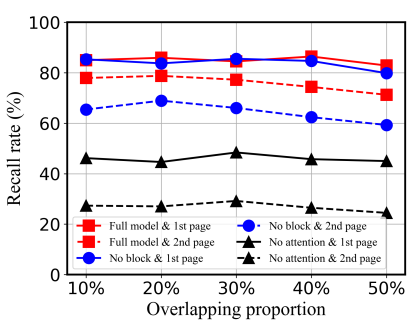

(c) Average recall rate

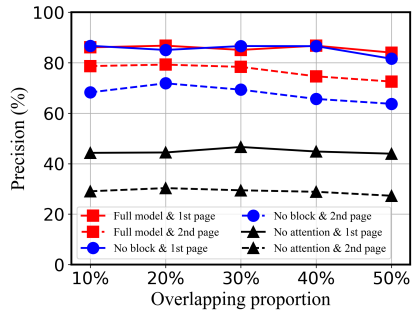

(b) Average precision

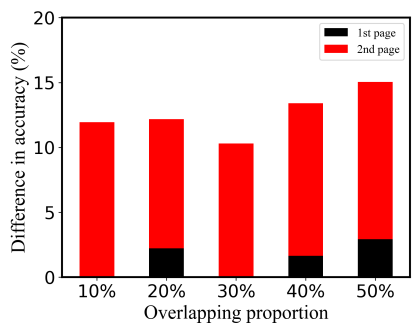

(d) Improvement on overall accuracy
Figure 5: Results of ablation analysis on BAPM

classification results especially for the second page (a more obvious improvement about $10 \%$ ). This is mainly because block division is the cornerstone of effectively exploiting the overlapping area, and this area plays a important role on the second page classification. In addition, Figure 5(d) displays the accuracy improvements on two pages, results of the first page (black bars) have almost no improvement under $10 \%$ proportion, but increase by $3 \%$ under $50 \%$ proportion, which means block division's effect will be more obvious on a larger overlapping area.

Towards the comparison between BAPM with and without multihead attention, results show that the attention mechanism doubles all metrics for the first page, and the improvement is even more obvious for the second page. Theoretically, BAPM would degenerate into a simple multi-label classifier like Multi-DF without attention, and block division attached to it would also become meaningless.

We display the attention distribution by visualizing the averaged vector over all vectors output by the attention layer (i.e., results of

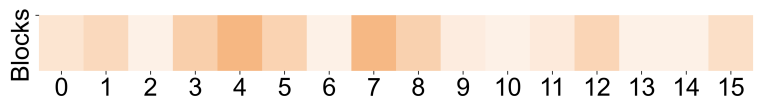

(a) Attention distribution of the first page tab

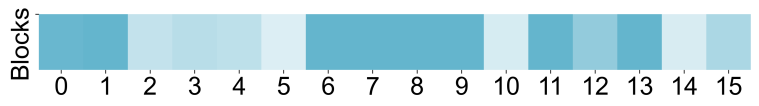

(b) Attention distribution of the second page tab

Figure 6: Attention distribution on blocks of two page tabs

masking the original representation with corresponding attention scores). We only take the vectors of correctly predicted samples into account for the reliability. Since there are two attention heads in our experiments, we get two averaged vectors finally as two tab-aware representation varieties on A.com and B.com. As we expect, the first head in Fig. 6(a) mainly focuses on the former part of A.com area, while the second head in Fig. 6(b) pays more attention on the latter part belonging to B.com. We notice that attention scores of the second head are mainly allocated on No. $6 \sim$ No.9 blocks within the overlapping area. This proves the importance of overlapping area acting as the beginning part of the second and also other following page tabs, same as previous findings. Note that No.1 and No.2 blocks get higher scores on both two heads and we guess it is possibly because similar anonymity circuits establishment generates similar characteristics on packet traces.

\subsection{Sensitivity Analysis}

Considering that the black-box nature of deep learning based methods is hard to intuitively display, we try to explain how dose BAPM contribute to its performance by tuning critical parameters and observing results in this section. In BAPM, the tab-aware feature representation is divided into several blocks to preserve local direction patterns of specific page tab. The block number $\mathbf{N}$ decides the granularity of attention mechanism when highlighting block relations. To find the best value of block number, we repeat the experiment in section 6.1 and the block number ranges from 1 to 512 . The overlapping proportion is set to be $50 \%$ since a large 


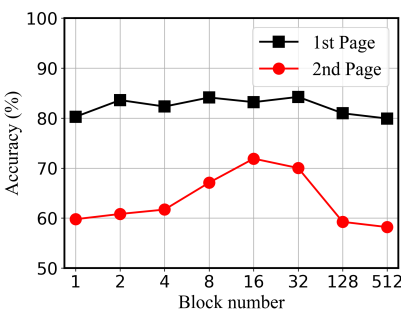

(a) Accuracy versus block numbers

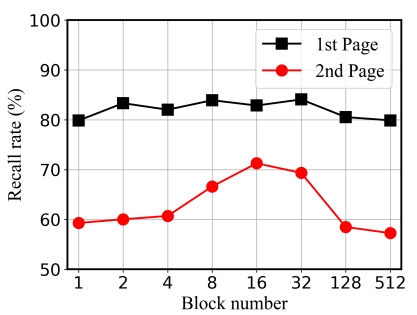

(c) Recall rate versus block numbers (b) Precision versus block numbers

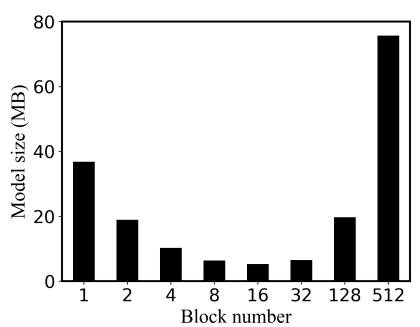

(d) Model size versus block numbers

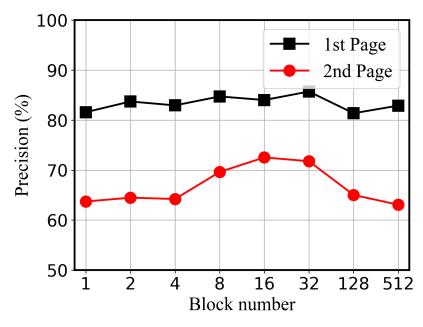

Figure 7: Results of sensitivity analysis on block number

overlapping area reflects block division's effectiveness more obviously. Accuracy, precision and recall rate are displayed in Fig. 7(a), Fig. 7(b) and Fig. 7(c), and there are two points in according with above theoretical analysis:

(1) All three evaluation metrics get better and then worse with block number increasing, indicating that too small or large block number both have negative effect. As discussed above, small number lets each block contain more mixed parts of different pages, and model has no idea which page tab this block has stronger connection with. On the contrary, reducing the block size would "purify" one block's content, but it's also harder to group blocks together through only a few features of small blocks. Besides, block division with proper block number improves results on two pages especially for the second one. Here the block number of 1 is equal to BAPM without block division in section 6.2 , and we detail the conclusion that effective block number ranges from 4 to 128 , with the value of 16 maximizing the effectiveness.

(2) The model size of BAPM shows a same trend as three evaluation metrics. Fig. 7(d) gives the model size in MB which firstly decreases and then rises again with the block number increasing. The tuning point of model size is in the almost same position as highest classification accuracy. Therefore, BAPM becomes most cost-effective when block number is around 16 with strong classification ability and low computational complexity.

\subsection{Results on Three-tab Packet Traces}

Three-tab classification results are shown as Table 4 with a uniform $10 \%$ overlapping proportion between two adjacent page tabs. BAPM achieves the best results on all three tabs, showing a better robustness when the tab number increases: results on the first tab are best since the beginning part is not destroyed, following two tabs are harder to classify especially the middle tab which is mixed with two tabs thus having the largest overlapping area. We notice that the accuracy of PSP-WF evidently declines compared with two-tab conditions, a possible reason is that three-tab traces have four overlapping points and traces will be wrongly split once PSP-WF wrongly predicts any one of points.

Table 4: Results on three-tab packet traces

\begin{tabular}{l|c|c|c|c}
\hline \multicolumn{2}{l|}{} & 1st page & 2nd page & 3rd page \\
\hline \multirow{3}{*}{ BAPM } & Acc & $\mathbf{9 1 . 3}$ & $\mathbf{6 3 . 5}$ & $\mathbf{7 4 . 7}$ \\
& Pre & $\mathbf{9 1 . 2}$ & $\mathbf{6 6 . 8}$ & $\mathbf{7 7 . 3}$ \\
& Rec & $\mathbf{9 1 . 1}$ & $\mathbf{6 3 . 3}$ & $\mathbf{7 4 . 6}$ \\
\hline \multirow{3}{*}{ PSP-WF } & Acc & 30.9 & 30.6 & 4.0 \\
& Pre & 36.2 & 34.4 & 3.7 \\
& Rec & 31.6 & 31.5 & 3.9 \\
\hline \multirow{3}{*}{ PSE-WF } & Acc & 23.0 & 15.6 & 18.9 \\
& Pre & 45.8 & 34.4 & 39.5 \\
& Rec & 22.0 & 15.1 & 17.6 \\
\hline \multirow{3}{*}{ Multi-DF } & Acc & 35.1 & 32.8 & 24.4 \\
& Pre & 36.6 & 32.7 & 25.0 \\
& Rec & 36.7 & 28.7 & 50.0 \\
\hline
\end{tabular}

Note that three page tabs are the maximum tab number which has been explored in practice among all multi-tab WF attacks. In reality, four-tab or more-tab traces may appear, but they are essentially same with three-tab traces in terms of the trace structure. As shown in Fig. 8, traces with more than two tabs all have three and only three types of tabs: the first tab with latter part overlapped, the last tab with front part overlapped, and the middle tab(s) with both front and latter part overlapped. Each page tab is mixed with two other tabs at most and no new case occurs when the tab number grows, thus the complexity does not increase. For our model, it's the tab type matters more instead of the tab number, and multi-tab fingerprints won't break as long as we adjust the BAPM architecture to make sure that one tab corresponds to one attention head. The only difference is that we need process longer packet traces during training and testing, at the expense of more model parameters and higher time consumption.

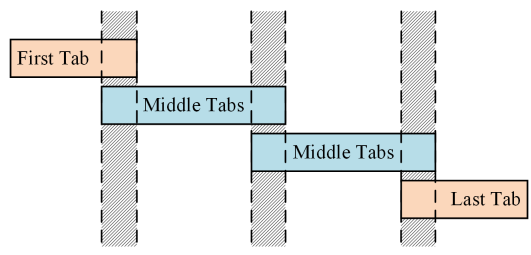

Figure 8: Three page tab types under more-tab scenarios

\subsection{Results on Open World Packet Traces}

We now evaluate the model performance under the open world setting. 2000, 4000 and 6000 open world websites are randomly selected from dataset of [27] as non-monitored websites, and the class number ratios of non-monitored websites to monitored ones 
are 40:1, 80:1 and 120:1, respectively. Packet traces of non-monitored websites appear on both training and testing sets which means that attackers can also take non-monitored traces to train their models. This assumption is called "the standard model under open world" in [19]. For each trace to classify, we firstly predict whether it belongs to a monitored website or not, and further predict the specific class if it is indeed a monitored trace.

Results under three scales of non-monitored websites are displayed as Table 5. All non-monitored websites are seen as an extra class, so evaluation metrics are calculated on 51 classes actually including fifty monitored and one non-monitored class. BAPM still outperforms other methods with results about $80 \%$ and $60 \%$ for the first and second tab. With non-monitored websites increasing, most metrics of four methods tend to decrease because a larger nonmonitored website set is more likely to have a trace with similar direction patterns to monitored ones, which is difficult to identify. Besides, the result difference between two tabs is particularly obvious under the open world setting.

Table 5: Results on open world dataset

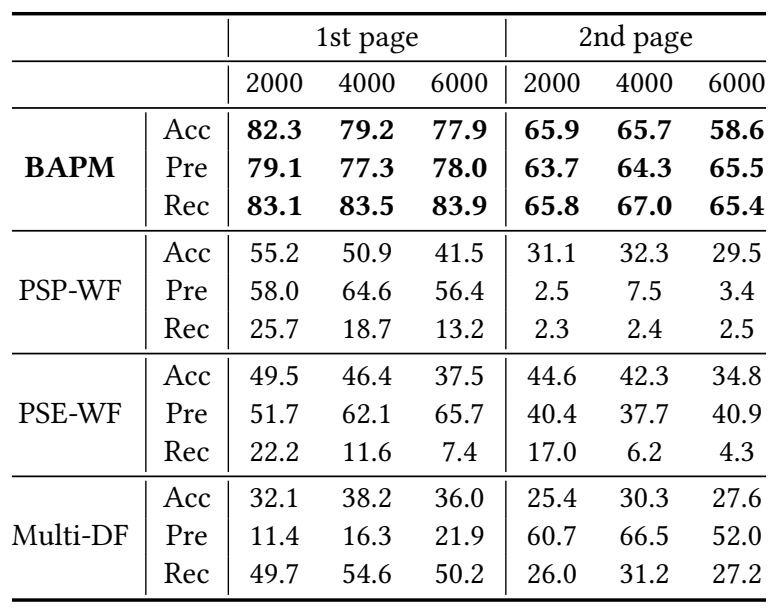

\section{DISCUSSION}

\subsection{Real world dataset analysis}

In this section, we use the real world dataset to verify that the effectiveness of BAPM will not be affected when attackers use manual traces to train the model and test it on real traces. We install a Tor proxy on a VPS host and drive a headless chrome browser to visit target websites through the proxy. An automatic script continuously visits the Alex top 50 websites recorded on march 18th, 2021. To build the training set with 10000 manual traces, it opens one website each time and captures 200 traces for each website. For the testing set with 1000 real traces, it opens the first page and waits few seconds, then opens the second one by starting a new thread and finally kills two threads in order. The loading time is set to 15 seconds uniformly. As [24] suggested, we clear browser cookies and wait few seconds before starting the next capture. We use the tool given in [24] to extract cell directions from traces as the model input. There are no ethical issues since we do not provide the proxy service on the data collection node for any other anonymous users.

Table 6: Results on real world multi-tab packet traces

\begin{tabular}{c|ccc|ccc}
\hline & \multicolumn{3}{|c|}{ 1st page } & \multicolumn{3}{c}{ 2nd page } \\
\hline & Acc & Pre & Rec & Acc & Pre & Rec \\
\hline BAPM & $\mathbf{9 3 . 2}$ & $\mathbf{9 3 . 5}$ & $\mathbf{9 2 . 9}$ & $\mathbf{8 2 . 9}$ & $\mathbf{8 4 . 9}$ & $\mathbf{8 3 . 2}$ \\
PSP-WF & 27.6 & 57.7 & 27.3 & 8.2 & 11.4 & 8.4 \\
PSE-WF & 70.7 & 81.4 & 70.3 & 70.5 & 80.7 & 70.1 \\
Multi-DF & 50.5 & 50.0 & 51.4 & 46.9 & 46.8 & 47.1 \\
\hline
\end{tabular}

As shown in Table 6. BAPM, PSE-WF and Multi-DF achieve even better results on real traces. We guess that different page tabs in real world may have some exclusive time slices to transmit data, while manually merging does not consider it and mix the data simply according to time stamps. The exception is that results of PSP-WF decline, which shows that overlapping points in manual and real traces may have different characteristics. In summary, real traces do not destroy the proved effectiveness of BAPM, and reasons mentioned in section 5.2 why we and attackers tend to choose manual traces as the training set are reasonable. The real world dataset is available at https://drive.google.com/drive/folders/ 1vfE34oYICpSnNMVvxfeVfcWzBooqAQfR?usp=sharing.

\subsection{Possible defenses analysis}

Like other WF attacks, injecting random traffic noise into original packet traces will destroy fingerprints extracted by BAPM. The noise may come from: (1) Simultaneous page tabs. In above experiments we focus on the multi-tab scenarios where users "successively" instead of "simultaneously" visit websites. "Successively" visiting appears in normal cases, while "simultaneously" visiting means users deliberately open extra tabs at the same time. Concurrent page tabs are completely mixed together as an interference confusing attackers. (2) Non-browsing behaviors during the web browsing generate irrelevant traffic which also disturbs original traces. Since the traffic noise is totally unpredictable, all WF works will be influenced to a certain extent.

A core principle of BAPM is trying to find local direction patterns of the specific website preserved by blocks, especially in overlapping area. When two tabs are involved, blocks we get may still well reflect a specific website's pattern. However, the random noise severely ruins original patterns in blocks under simultaneous tabs or non-browsing behaviors, making block relations more difficult to discover. We performed a simple test by adding the third and fourth tab into the overlapping area of two-tab traces as the random noise. Results show that after adding the fourth tab (i.e., the bandwidth overhead reached $100 \%$ compared with only two tabs opened), the accuracy for the second tab drops to almost equal with random decision, meaning that multi-tab fingerprints are no longer effective. Therefore, keeping the influence of simultaneous tabs or non-browsing behaviors during the browsing process will be helpful in defending against potential multi-tab WF attacks. 


\section{CONCLUSION}

In this paper, we propose a deep learning WF attacking model named BAPM against multi-tab browsing behavior. BAPM transforms cell direction sequences including overlapping and nonoverlapping area into blocked tab-aware representations, which help to avoid information confusion even lost under both tabs and blocks level. Besides, we apply the multi-head attention mechanism to the blocks aggregation on the same specific website and generate a representation variety on each attention head. As a result, multiple website labels are simultaneously predicted according to corresponding representation varieties, and attackers do not need to accurately predetermine website locations in raw packet traces. BAPM outperforms several well-known multi-tab WF attacks on the comparison experiment, and the model design effectiveness is also explained through comprehensive analysis.

We believe that this work makes a step forward on the feasibility of WF attacks on Tor, which reminds users that their privacy is possible to be threatened even under more realistic multi-tab scenarios. Therefore, we also introduce some possible defenses against our model and discuss how does the fingerprint break with defenses. It will be safer for users to consciously make some noise to protect themselves if they do not use proposed countermeasures. Some extended scenarios such as the fingerprints drift over time or how to design multi-tab WF models against countermeasures can be researched in the future.

\section{ACKNOWLEDGMENTS}

This work is supported by The National Key Research and Development Program of China (No.2020YFB1006100, No.2020YFE0200500 and No.2018YFB1800200), Key research and Development Program for Guangdong Province under grant No.2019B010137003 and the Strategic Priority Research Program of Chinese Academy of Sciences, grant No.XDC02040400. We would also like to thank reviewers for their valuable comments to improve this paper.

\section{REFERENCES}

[1] 2021. Tor, 2021. https://www.torproject.org/

[2] Kota Abe and Shigeki Goto. 2016. Fingerprinting Attack on Tor Anonymity using Deep Learning. In Proceedings of the APAN - Research Workshop.

[3] Sanjit Bhat, David Lu, Albert Kwon, and Srinivas Devadas. 2019. Var-CNN: A DataEfficient Website Fingerprinting Attack Based on Deep Learning. Proceedings on Privacy Enhancing Technologies (Oct. 2019), 292-310. 10.2478/popets-2019-0070

[4] Xiang Cai, Xin Cheng Zhang, Brijesh Joshi, and Rob Johnson. 2012. Touching from a distance: website fingerprinting attacks and defenses. In the ACM Conference on Computer and Communications Security, CCS, Raleigh, NC, USA. 605-616. https://doi.org/10.1145/2382196.2382260

[5] Kyunghyun Cho, Bart van Merrienboer, Çaglar Gülçehre, Dzmitry Bahdanau, Fethi Bougares, Holger Schwenk, and Yoshua Bengio. 2014. Learning Phrase Representations using RNN Encoder-Decoder for Statistical Machine Translation. In Proceedings of the Conference on Empirical Methods in Natural Language Processing, EMNLP, Doha, Qatar. 1724-1734. https://doi.org/10.3115/v1/d14-1179

[6] Weiqi Cui, Tao Chen, Christian Fields, Julianna Chen, Anthony Sierra, and Eric Chan-Tin. 2019. Revisiting Assumptions for Website Fingerprinting Attacks. In Proceedings of the ACM Asia Conference on Computer and Communications Security, AsiaCCS, Auckland, New Zealand. 328-339. https://doi.org/10.1145/ 3321705.3329802

[7] Roger Dingledine, Nick Mathewson, and Paul F. Syverson. 2004. Tor: The SecondGeneration Onion Router. In Proceedings of the 13th USENIX Security Symposium, San Diego, CA, USA. 303-320. http://www.usenix.org/publications/library/ proceedings/sec04/tech/dingledine.html

[8] Xiaodan Gu, Ming Yang, and Junzhou Luo. 2015. A novel Website Fingerprinting attack against multi-tab browsing behavior. In 19th IEEE International Conference on Computer Supported Cooperative Work in Design, CSCWD, Calabria, Italy. 234-239. https://doi.org/10.1109/CSCWD.2015.7230964
[9] Jamie Hayes and George Danezis. 2016. k-fingerprinting: A Robust Scalable Website Fingerprinting Technique. In 25th USENIX Security Symposium, USENIX Security, Austin, TX, USA. 1187-1203. https://www.usenix.org/conference/ usenixsecurity $16 /$ technical-sessions/presentation/hayes

[10] Andrew Hintz. 2002. Fingerprinting Websites Using Traffic Analysis. In Privacy Enhancing Technologies, Second International Workshop, PET, San Francisco, CA, USA (Lecture urls in Computer Science, Vol. 2482). 171-178. https://doi.org/10. 1007/3-540-36467-6 13

[11] Marc Juárez, Sadia Afroz, Gunes Acar, Claudia Díaz, and Rachel Greenstadt. 2014. A Critical Evaluation of Website Fingerprinting Attacks. In Proceedings of the ACM SIGSAC Conference on Computer and Communications Security, Scottsdale, AZ, USA. 263-274. https://doi.org/10.1145/2660267.2660368

[12] Shuai Li, Huajun Guo, and Nicholas Hopper. 2018. Measuring Information Leakage in Website Fingerprinting Attacks and Defenses. In Proceedings of the ACM SIGSAC Conference on Computer and Communications Security, CCS, Toronto, ON, Canada. 1977-1992. https://doi.org/10.1145/3243734.3243832

[13] Marc Liberatore and Brian Neil Levine. 2006. Inferring the source of encrypted HTTP connections. In Proceedings of the 13th ACM Conference on Computer and Communications Security, CCS, Alexandria, VA, USA. 255-263. https://doi.org/10. 1145/1180405.1180437

[14] Liming Lu, Ee-Chien Chang, and Mun Choon Chan. 2010. Website Fingerprinting and Identification Using Ordered Feature Sequences. In Computer Security ESORICS, 15th European Symposium on Research in Computer Security, Athens, Greece (Lecture urls in Computer Science, Vol. 6345). 199-214. https://doi.org/10. 1007/978-3-642-15497-3_13

[15] Andriy Panchenko, Fabian Lanze, Jan Pennekamp, Thomas Engel, Andreas Zinnen, Martin Henze, and Klaus Wehrle. 2016. Website Fingerprinting at Internet Scale. In 23rd Annual Network and Distributed System Security Symposium, NDSS, San Diego, California, USA. http://wp.internetsociety.org/ndss/wpcontent/uploads/sites/25/2017/09/website-fingerprinting-internet-scale.pdf

[16] Andriy Panchenko, Lukas Niessen, Andreas Zinnen, and Thomas Engel. 2011. Website fingerprinting in onion routing based anonymization networks. In Proceedings of the 10th annual ACM workshop on Privacy in the electronic society, WPES, Chicago, IL, USA. 103-114. https://doi.org/10.1145/2046556.2046570

[17] Aida Ramezani, Amirhossein Khajehpour, and Mahdi Jafari Siavoshani. 2020. On Multi-Session Website Fingerprinting over TLS Handshake. CoRR abs/2009.09284 (2020). arXiv:2009.09284 https://arxiv.org/abs/2009.09284

[18] Vera Rimmer, Davy Preuveneers, Marc Juarez, Tom Van Goethem, and Wouter Joosen. 2017. Automated Website Fingerprinting through Deep Learning. In Network and Distributed System Security Symposium.

[19] Payap Sirinam, Mohsen Imani, Marc Juarez, and Matthew Wright. 2018. Deep Fingerprinting: Undermining Website Fingerprinting Defenses with Deep Learning. In Proceedings of the ACM SIGSAC Conference on Computer and Communications Security, CCS, Toronto, ON, Canada. 1928-1943. https://doi.org/10.1145/3243734. 3243768

[20] Payap Sirinam, Nate Mathews, Mohammad Saidur Rahman, and Matthew Wright. 2019. Triplet Fingerprinting: More Practical and Portable Website Fingerprinting with $\mathrm{N}$-shot Learning. In Proceedings of the ACM SIGSAC Conference on Computer and Communications Security, CCS, London, UK. 1131-1148. https://doi.org/10. 1145/3319535.3354217

[21] Ashish Vaswani, Noam Shazeer, Niki Parmar, Jakob Uszkoreit, Llion Jones, Aidan N. Gomez, Lukasz Kaiser, and Illia Polosukhin. 2017. Attention is All you Need. In Annual Conference on Neural Information Processing Systems, Long Beach, CA, USA. 5998-6008. https://proceedings.neurips.cc/paper/2017/hash/ 3f5ee243547dee91fbd053c1c4a845aa-Abstract.html

[22] Christian von der Weth and Manfred Hauswirth. 2013. DOBBS: Towards a Comprehensive Dataset to Study the Browsing Behavior of Online Users. In IEEE/WIC/ACM International Conferences on Web Intelligence, WI, Atlanta, GA, USA. 51-56. https://doi.org/10.1109/WI-IAT.2013.8

[23] Meiqi Wang, Yanzeng Li, Xuebin Wang, Tingwen Liu, and Muqian Chen. 2020. 2ch-TCN: A Website Fingerprinting Attack over Tor Using 2-channel Temporal Convolutional Networks. In IEEE Symposium on Computers and Communications (ISCC).

[24] Tao Wang, Xiang Cai, Rishab Nithyanand, Rob Johnson, and Ian Goldberg. 2014. Effective Attacks and Provable Defenses for Website Fingerprinting. In 23rd USENIX Security Symposium. San Diego, CA, 143-157. https://www.usenix.org/ conference/usenixsecurity14/technical-sessions/presentation/wang_tao

[25] Tao Wang and Ian Goldberg. 2013. Improved website fingerprinting on Tor. In Proceedings of the 12th annual ACM Workshop on Privacy in the Electronic Society, WPES, Berlin, Germany. 201-212. https://doi.org/10.1145/2517840.2517851

[26] Tao Wang and Ian Goldberg. 2016. On Realistically Attacking Tor with Website Fingerprinting. Proc. Priv. Enhancing Technol. 2016, 4 (2016), 21-36. https: //doi.org/10.1515/popets-2016-0027

[27] Tao Wang and Ian Goldberg. 2017. Walkie-Talkie: An Efficient Defense Against Passive Website Fingerprinting Attacks. In 26th USENIX Security Symposium, Vancouver, BC. 1375-1390. https://www.usenix.org/conference/usenixsecurity17/ technical-sessions/presentation/wang-tao 
[28] Kelvin Xu, Jimmy Ba, Ryan Kiros, Kyunghyun Cho, Aaron C. Courville, Ruslan Salakhutdinov, Richard S. Zemel, and Yoshua Bengio. 2015. Show, Attend and Tell: Neural Image Caption Generation with Visual Attention. In Proceedings of the 32nd International Conference on Machine Learning, ICML, Lille, France, Vol. 37. 2048-2057. http://proceedings.mlr.press/v37/xuc15.html

[29] Yixiao Xu, Tao Wang, Qi Li, Qingyuan Gong, Yang Chen, and Yong Jiang. 2018 A Multi-tab Website Fingerprinting Attack. In Proceedings of the 34th Annual
Computer Security Applications Conference, ACSAC, San fuan, PR, USA. 327-341. https://doi.org/10.1145/3274694.3274697

[30] Zhongliu Zhuo, Yang Zhang, Zhi-Li Zhang, Xiaosong Zhang, and Jingzhong Zhang. 2018. Website Fingerprinting Attack on Anonymity Networks Based on Profile Hidden Markov Model. IEEE Trans. Inf. Forensics Secur. 13, 5 (2018), 1081-1095. https://doi.org/10.1109/TIFS.2017.2762825 